19 Revue d'histoire du XIXe siècle

Société d'histoire de la révolution de 1848 et des

révolutions du XIXe siècle

$60 \mid 2020$

Relire les expériences de guerre franco-allemandes (1870-1871)

\title{
Euvres de Jean Jaurès, tome 11, Voici le XXe siècle 1905-1907, édition établie par Vincent DUCLERT
}

\section{Raymond Huard}

\section{(2) OpenEdition}

Journals

Édition électronique

URL : https://journals.openedition.org/rh19/6962

ISSN : $1777-5329$

Éditeur

La Société de 1848

Édition imprimée

Date de publication : 1 juin 2020

Pagination : 278-280

ISSN : 1265-1354

Référence électronique

Raymond Huard, « Fuvres de Jean Jaurès, tome 11, Voici le xxe siècle 1905-7907, édition établie par Vincent DUCLERT », Revue d'histoire du XIXe siècle [En ligne], 60 | 2020, mis en ligne le 01 juin 2020, consulté le 10 janvier 2023. URL : http://journals.openedition.org/rh19/6962

Ce document a été généré automatiquement le 10 janvier 2023.

Tous droits réservés 


\title{
CEuvres de Jean Jaurès, tome 11, Voici le $\mathrm{XX}^{e}$ siècle 1905-1907, édition établie par Vincent DUCLERT
}

\author{
Raymond Huard
}

\section{RÉFÉRENCE}

CEuvres de Jean Jaurès, tome 11, Voici le Xxe siècle 1905-1907, édition établie par Vincent DUCLERT, Paris, Fayard et société d'Études jaurésiennes, 2019, 683 p., $35 €$.

1 La publication des CEuvres de Jean Jaurès, commencée il y a maintenant vingt ans, se poursuit, grâce à la persévérance de la Société d'Études jaurésiennes, avec la parution de ce tome 11, qui couvre les années 1906 et $1907^{1}$. Cette période est importante puisqu'elle est celle de l'achèvement de la Séparation, de la fin du Bloc des Gauches et des élections de 1906, de la montée d'un mouvement ouvrier en lutte avec le gouvernement radical, de la révolte du Midi en 1907, des suites de la révolution de 1905 en Russie et du début de la mainmise française sur le Maroc alors que la menace de guerre européenne commence à se profiler. La production de Jaurès pendant ces années, qu'il s'agisse d'articles de La Dépêche ou de L'Humanité, de discours aux congrès socialistes nationaux (Nancy, août 1906) ou internationaux (Stuttgart, août 1907), plus rarement de lettres, est d'une telle abondance (plus de 500 interventions au total) que l'ouvrage ne peut fournir qu'un choix, déjà très substantiel, de 95 textes. Trois grands discours parlementaires ${ }^{2}$ occupent d'ailleurs $20 \%$ du volume. Comme précédemment, ces textes sont classés en sept grands chapitres pour couvrir les principaux thèmes, à l'intérieur desquels ils sont rangés par ordre de date. Il n'est pas facile d'ailleurs de classer rigoureusement ces textes, Jaurès évoquant souvent plusieurs thèmes dans une seule intervention. Ces textes sont éclairés par des notes précises, en particulier sur les personnages mentionnés. Il aurait sans doute été nécessaire d'éclairer un peu plus certains événements historiques dont il est question. 
2 Jaurès est certes soucieux de voir s'achever les réformes antérieures, la Séparation en particulier. Il souhaite une application ferme, mais souple de la loi face à la "grande folie » de l'encyclique papale, contraire à l'opinion du clergé français qui était disposé à appliquer la loi. Mais il ne veut pas que cette question occulte l'essentiel, c'est-à-dire la nécessité de réformes sociales, et il presse les gouvernements de les mener à bien, que ce soit les retraites ouvrières ou l'impôt sur le revenu. Il doit constater malheureusement que, contrairement à ses espoirs, celles-ci s'enlisent, y compris sous le gouvernement radical de Clemenceau. Or le mouvement ouvrier est en croissance et se manifeste par des grèves comme celle, remarquée, de Fougères ou celles qui suivent la catastrophe minière de Courrières. Vis-à-vis de celui-ci, Jaurès adopte une attitude remarquablement compréhensive. Contrairement à ceux qui interprètent négativement la Charte d'Amiens, le plus important à ses yeux est que le congrès d'Amiens a fixé des objectifs qui sont très proches de ceux des socialistes. Il considère avec sympathie la CGT parce qu'elle réalise l'unification des forces syndicales, ne veut pas réduire le syndicalisme à un rôle uniquement corporatif, soutient l'extension du syndicalisme aux instituteurs (tout en désapprouvant cependant qu'ils puissent faire grève). Il s'oppose avec vigueur à la politique de répression antisyndicale en des termes qui sont parfois d'une brûlante actualité dans les conflits d'aujourd'hui. Il est amené plusieurs fois à définir sa vision de la révolution sociale qu'il voudrait pacifique car il est convaincu que "bientôt presque toute la classe ouvrière sera conquise au socialisme » (14 août 1906). Il n'exclut pas d'exproprier légalement les capitalistes pour créer " une humanité où le travail sera souverain ", grâce à " une forme collective et sociale de la propriété ». Dans l'impôt sur le revenu, qu'il défend avec force bien qu'il soit conscient que celui-ci n'est pas un pas en avant vers le socialisme, il voit notamment l'occasion de dégrever efficacement la petite propriété paysanne. En 1907, Jaurès élu du Midi ne peut que sympathiser avec les difficultés des viticulteurs, mais il ne veut pas non plus que les concessions qui seraient faites au Midi mettent en danger l'unité nationale et semble un peu embarrassé. Si la nationalisation de la vigne qu'il propose apparait peu réaliste, d'autres suggestions sont plus classiques (développement de la coopération, irrigation, essor industriel grâce à la houille blanche).

Dans le domaine international, trois sujets dominent sa réflexion: le rapport avec l'Allemagne, les conséquences de l'alliance russe, et l'intervention au Maroc. Face aux attaques portées contre lui sur le terrain du patriotisme, il défend une attitude propre à rallier aussi les socialistes allemands. En cas de menace de conflit, on doit recourir à l'arbitrage international, thème majeur de sa pensée à l'époque. Si celui-ci échouait, un mouvement de masse international doit empêcher la guerre. Au cas où un pays serait attaqué cependant, la défense nationale est de rigueur. L'expérience de 1914 montrera la fragilité de cette construction. Jaurès, toujours méfiant quant aux conséquences de l'alliance franco-russe, craint que les emprunts russes en France ne servent à consolider le pouvoir autocratique face à son opposition. Enfin, il perçoit fort bien les visées profondes des débuts de l'intervention française au Maroc, malgré les dénégations officielles. Ici encore, le recours à une gestion internationale du cas marocain lui paraît nécessaire. Si l'on admire toujours en lisant ces pages la prodigieuse culture de Jaurès, sa connaissance très approfondie de dossiers complexes (sur l'impôt sur le revenu par exemple), son talent oratoire et sa capacité de repartie, une autre caractéristique est aussi frappante, c'est, à partir d'une situation donnée, sa capacité à se projeter dans l'avenir en imaginant le scénario de ce qui pourrait arriver. Si ses 
prévisions sont loin de se réaliser toujours, elles révèlent un homme résolument tourné vers l'avenir, envisagé volontiers de façon optimiste.

\section{NOTES}

1. Deux tomes qui couvriront les années 1907 à 1914 restent encore à paraître.

2. Polémique avec Clemenceau, avec Briand, et intervention sur la crise du Midi en 1907. 